\title{
Hand Kinesics in Indian Sign Language using NLP Techniques with SVM Based Polarity
}

\author{
Haren Amal, Reema Ann Reny, Boppuru Rudra Prathap
}

\begin{abstract}
With the advent of new technology every year, human beings continue to make clever innovations to benefit not only themselves but also those with some kind of impairment. Communication is carried out by talking to each other for regular people, but people who are deaf interact with each other through sign language. Taking this problem into account, we are proposing a methodology that allows to ease the communication with each other by translating speech into sign language. This paper explains a methodology that translates speech into the corresponding Indian Sign Language (ISL). In India, it is spoken in almost 28 different languages. So, language has always been a problem. Thus, we have come with a project just for India in which the person can communicate with the app in any Indian language they know, and it will convert it into Indian Sign Language. This is applicable to not just literate but also illiterate people across India. The idea is to take the speech input and translate to text, which will then undergo text-pre-processing using NLP for better analysis and will be connected to the HamNoSys data for the generation of sign languages. The polarity detection will also be included. It is implemented using the SVM algorithm for Sentimental Analysis. Thus, the main objective of this project is to develop a useful project which can be used to capture the whole vocabulary of Indian Sign Language (ISL) and provide access to information and services to mute people in ISL.
\end{abstract}

Keywords: Indian Sign Language, Speech Recognition, Python, Natural Language Processing, Support Vector Machine algorithm, Polarity Detection.

\section{INTRODUCTION}

In India, according to the 2011 census, there are roughly 1.3 million individuals with "hearing impairment" distinction that to numbers from India's National Association of the Deaf, which estimates that eighteen million folks. They use this language as a medium of communication, however, people don't need to be conscious of the language. This will produce an issue in human activity with them. In the past few years, analysis has increased appropriately in relevance to the employment of computers to acknowledge. Technology is dynamic quickly and is rising the approach the globe

Revised Manuscript Received on April 25, 2020.

* Correspondence Author

Haren Amal*, B. Tech in Computer Science and Engineering at CHRIST (Deemed to be University) India.

Reema Ann Reny, B.Tech in Computer Science and Engineering at CHRIST (Deemed to be University) India.

Boppuru Rudra Prathap, Assistant Professor and Research Scholar, Computer Science and Engineering at CHRIST (Deemed to be University) India.

(C) The Authors. Published by Blue Eyes Intelligence Engineering and Sciences Publication (BEIESP). This is an open access article under the CC BY-NC-ND license (http://creativecommons.org/licenses/by-nc-nd/4.0/) operates; it's unfolded the barriers for those who are hard hearing. Many people are attempting to create systems which will impact the approach for the mute people communicate through the employment of machine learning, AI, image process and pattern matching techniques, so rising the standard of life through translating the spoken speech to text and so the sign language. With the help of sign language, these individuals can communicate among them and with normal individuals. Sign language may be a language employed by hard speaking and hard hearing those that uses visual sign patterns to express that means. It is the mixture of hand shapes, movement and position of hands, arms or body, and also includes facial expressions. There are around $700,000,000$ deaf or mute individuals worldwide (World Health Organization). There also exist 143 different language dialects that uniquely define the language. All-round the world the sign languages used is going to be different. For instance, for the USA it's the American Sign Language (ASL); Britain sign language (BSL) for Britain; and Indian sign language (ISL) is employed in India for conveying thoughts with other people. During this analysis paper, we are going to be implementing the Indian language (ISL) with the assistance of the speech input.

The major drawback faced by the Indian sign language Interpreter was the anomaly within the language community. The words that required to be enclosed in sign language dictionary were limited. Indian sign language analysis and coaching Centre developed "The Indian Sign Language" (ISL) dictionary. It consisted of 3,000 words which were in Hindi and English languages respectively and along with the corresponding illustration of the corresponding signs. This was created to get rid of the anomaly throughout the nation.

\subsection{Novelty of the Proposed Work}

This analysis paper proposes a system especially for India, to assist individuals simply communicate with mute people using any of the Indian languages as input and provides suitable Indian sign language (ISL). ISL may be a complete linguistic communication with its descriptive linguistics and syntax. During this method, it's vital to touch upon an honest methodology considering the most aspects. This reality becomes additionally relevant once it involves users who have any reasonable incapacity.

The ISL translation system uses a microphone to record voice (from normal people) which may be understood by the applying. The technical specifications of this project are supported Google API, changing the speech received to text using speech to text API, which can be translated to English text using googletrans Python library that enforced Google translate API.

Published By:

Blue Eyes Intelligence Engineering DOI: 10.35940/ijeat.D8483.049420

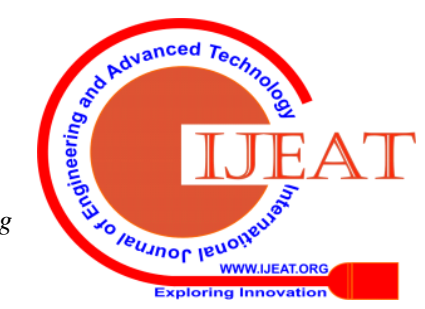




\section{Hand Kinesics in Indian Sign Language using NLP Techniques with SVM Based Polarity}

Then using the semantics of natural language process to breakdown the text into perceivable items. It then translates the audio speech to the text that follows ISL grammar. The next aim would be to determine the polarity of the text produced. This needs a Machine Learning algorithm as a part. The machine learning algorithmic program which will be used here is the Support Vector Machine (SVM) algorithm for polarity detection. Support vector machines (SVMs) are a group of supervised learning ways used for classification, regression and outlier detection. Thus, we can study about the sentimental analysis of the given speech text. For the predefined sign language, data sets are used. The graphical user interface application is showing and causing the message to the receiver. Thus, it focuses to make the communication with normal individuals and the hard hearing/mute individuals easier.

\section{LITERATURE SURVEY}

This part explains about the issues and background of the Audio to Sign Language systems and its various existing algorithms. Many systems contribute to different communities of mute people. As we have discussed before, there are different communities of Sign Languages and there are many papers related to different Sign Language. Here we have considered different papers that use different methodologies for implementation and for different communities.

Research by M. Jerin Jose et.al. (2013) [1] have contributed to the Rule-based Text Matching. Here, it uses context-dependent continuous Hidden Markov Models (HMMs) designed using decision tree state agglomeration. There are 153 translation rules in the rule-based translation module and therefore the translation method is meted out in 2 steps: within the initiative, each word is mapped to 1 or many syntactic- pragmatic tags. Other studies include V. López-Ludeña et.al. (2013) [2] who have contributed using applied math machine translation. In this system, the natural language translation module converts a word sequence into a proof sequence. Malaysian Deaf People. The Mel Frequency Cepstral Coefficients (MFCC) rule was accustomed to implement this method. Using the Digital Signal Processing (DSP), the sound recorded from its time domain to the frequency domain is regenerated. Also, vector quantization is employed to perform speech recognition that provides an efficient matching technique [3].

Khalid Khalil El-Darymli et.al. (2006) [4] have developed for the American Sign Language (ASL). This is done exploitation the Sphinx 3.5 software. This system was used for manipulating American Sign Language (ASL) within the code. This was achieved by following the Signed English (SE) model. Sphinx was used, that could be a fairly giant program that gives tons of tools and knowledge. Sphinx 3 works best on continuous speech and huge vocabulary and has been tested.

According to a research by Sandeep Kaur et.al. (2015) [17] have proposed a system that generates HamNoSys supported ISL similar to words. The notations generated square measure per the signs employed in India. The input is given to JA SIGML URL APP and therefore the corresponding HamNoSys notations are displayed by the
Work done by Oi Mean Foong et.al. (2009) proposes for

avatar. Also, other research done by Parteek Kumar et.al. (2018) [7] provides a visible gesture to convey thoughts. It's developed with the assistance of PHP and net Graphics Library (WebGL) technology for the 3D animation. There's a wordbook that stores the multilingual information of words; each in Hindi and English language.

Other researchers' R. Manoharan et.al. (2014) [18] have written on the Smart Phone-Based whose goal was to make not simply a speech recognition for translating to ISL, however conjointly a semantic analysis was done with the assistance of the Google voice. There's conjointly a gesture sequence generation a gesture taking part in the method that they need to target a lot of on. Regardless of the gesture sequence generator that can turn out, the animated human agent can perform the acceptable hand signs. The gesture sequence will learn from the user if the speech isn't properly recognized or if the words aren't found within the lexicon.

Research from Pooja Balu Sonawane et.al. (2018) have contributed to design an independent communication system for people who are mute or for people who are hard hearing. It uses the vision-based system. The input used here are the alphabets and numerals. It is then converted into equivalent sign code which will be displayed on a screen. It displays the equivalent symbols for alphabets because the output in line with Raspberry pi Board makes this method compact and simply moveable [19].

Taner Arsan et.al. (2015) [9] have written on a motion capture system is employed for sign language conversion and a voice recognition system for voice conversion. The concept is that it captures the voice from the user and displays the output in the form of motioned images or videos. It records the acoustic audio signals that then convert to text and later to the gif files.

An Integrated Two Way ISL (Indian Sign Language) Translation System was contributed by M. Suresh Anand et.al. (2013) [10] which proposes a system to assist individuals to communicate while not using any refined device, however, it instead uses a camera and a microphone to implement the system. Once obtaining vectors feature extraction state, the next step will be performed which is pattern matching. This is done by comparison existing information. The understood symbols (meaning - words) will be translated into text data.

Another approach by Philippe Dreuw et.al. (2007) [11] discusses based on Bayes' decision rule. Each word model consists of one to a few pseudo phonemes. This will be sent for modelling the typical word length which was seen during training. Every phone is sculptured by hidden Markov model (HMM). It follows a 3-state left-to-right with 3 separate Gaussian mixtures (GMM) and a globally pooled variance matrix.

A. Gayathri et.al. (2017) [12] contributed using Android Environment which uses Speech-to-sign technology and VRS permits audible language translation on good phones with language and application has characters feature in mobile while not dialing range uses a technology. This technology takes the input not only in the form of speech but also takes the input in written form. It later interprets it to sign language with video.

\section{Published By:}

Blue Eyes Intelligence Engineering 
Another research by Veronica L'opez- Lude'na et.al. (2011) contributed to the Methodology for developing a Speech into Sign Language Translation System. It proposes a methodology for developing a user-centered strategy [16].

Similarly, Gaurangi Patil et.al. (2014) [13] have contributed to the Sentiment Analysis Using Support Vector Machine (SVM). Sentiment analysis is a subtopic of NLP and deals with determining the opinion and subjectivity in a text, which has many applications. It specifies whether the given data is positive, negative or neutral. This helps the machine to analyze the subjective content provided and can thus conclude it. It uses the SVM algorithm due to its easiness to use and the accuracy obtained.

\section{METHODOLOGY}

The main goal here is to implement speech to Indian Sign Language (ISL) to help the mute people in India to communicate easily with other people. To perform this, we need to divide the entire model into 5 steps or sub-objectives which are as follows:

1. Speech Recognition

2. Text Pre-processing using NLP

3. Polarity detection using Sentimental Analysis

4. Dictionary-based Machine Translation

5. Creating the user interface

The input will be provided as live speech or audio which will be sent for text pre-processing using the semantics of NLP. This text will be sent to under the Machine Learning algorithm for Polarity Detection and the corresponding Sentimental analysis will be obtained. After this process, the processed text will be obtained as the ISL text and the avatar will display the sign language appropriately.

The Natural Language Processing (NLP) techniques are also used in the implementation process. This is mainly used for the text pre-processing module, obtained after recognizing the speech. The techniques used here are stemming, tokenizing, lemmatization and creation of bag-of-words. Sentiment analysis uses the natural language processing techniques for text analysis to systematically identify, extract, quantify, and study the subjective information. In this project, we will be using the SVM algorithm. This is used to check if the provided speech input is a positive, negative or neutral statement. SVM is one of the most popularly used supervised machine learning algorithms for textual polarity detection.

\subsection{Proposed system}

Figure.1 explains the overall procedure of the proposed system. The proposed system consists of the following steps: i. Speech input ii. Noise removal iii. Speech Recognition iv. Text-Pre-processing v. Sentimental Analysis using SVM algorithm vi. Dictionary-based Machine Translation vii. Recognized Sign Symbol.

\section{i. $\quad$ Speech Input}

The input speech will be taken with the help of a Personal Device Assist (PDA); in this case, we will be using the microphone as the hardware for speech input. For better quality, the speech will also be sent for noise removal, which is the next step. This is achieved through the use of Python library PyAudio along with some coding.

\section{ii. $\quad$ Noise Removal}

The speech that was taken via a microphone will be sent to the next stage, for noise removal. We will be victimization the Filtering Techniques to filter the unwanted sounds or noise within the background, so improve its potency. These techniques embrace Spectral subtraction methodology through subtraction of an estimate of the common noise spectrum that is obtained from noisy signal spectrum. Adjusted noise cancellation is additionally an approach used for noise reduction in the speech signal. As the received signal is unceasingly corrupted by noise where each received signal and noise signal each change unceasingly, then this arises the requirement of adaptive filtering.

\section{iii. Speech Recognition}

Speech Recognition consecutive step would be to acknowledge the speech input, which might be achieved with the assistance of speech_recogniton library in Python. It identifies the language that we tend to speak using the Google Speech to Text API and also the corresponding text is going to be displayed in the language spoken by the user. The Google language codes are offered for various Indian languages which might be integrated with the code so that multiple languages are often detected and recognized by the system.

\section{iv. Text-Pre-processing}

As shown in the figure, using the syntax and semantics of Natural Language Processing, the text will be broken down into smaller understandable pieces. This involves Lexical analysis, tokenizing and stemming. To pre-process your text merely means that to bring your text into a kind that's certain and analyzable for your task. To grasp the meaning of the text, it's referred to as linguistics. A completely adequate natural language semantics would need a whole theory of however individuals assume and communicate concepts. One such approach uses the alleged"logical form," which may be an illustration of meaning supported the acquainted predicate and lambda calculi. In this way, the text will be processed more easily for the next step for rule-based text matching.

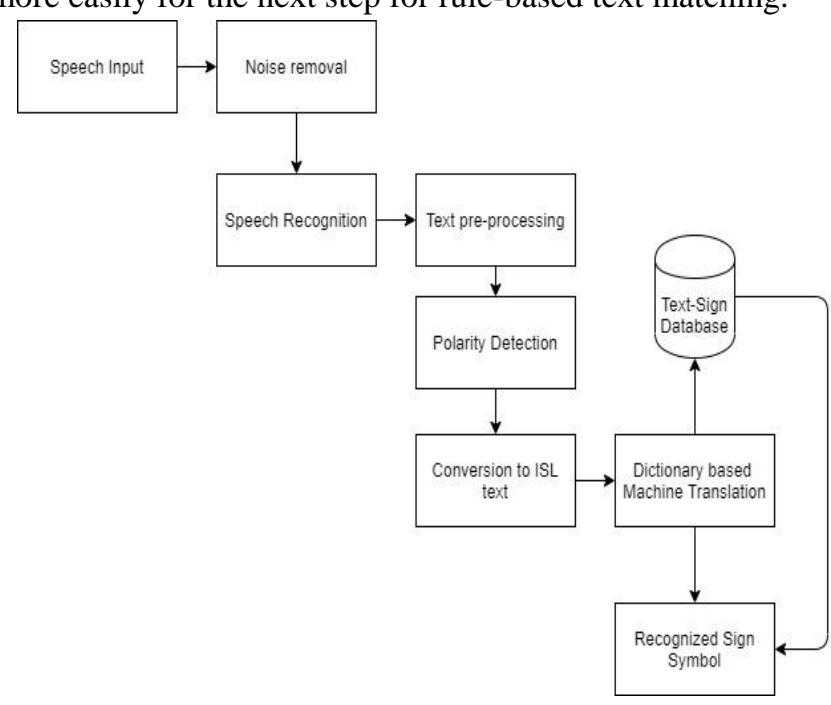

Figure.1 Proposed architecture

\section{v. Sentimental Analysis using the SVM algorithm}

Support Vector Machine (SVM) was used for the classification purposes.

Published By:

Blue Eyes Intelligence Engineering 


\section{Hand Kinesics in Indian Sign Language using NLP Techniques with SVM Based Polarity}

It accustomed to classify the texts as positives or negatives. SVM is a great method that can be used for text classification because of its benefits like its potential to handle massive options.

One of the pros is that SVM is robust once there's a sparse set of examples and additionally, as a result of most of the problems are linearly dissociable. Support Vector Machine has shown promising ends up in sentiment analysis.

\section{vi. Dictionary-based Machine Translation}

Using a Rule-based classification algorithm, the text is analyzed and appropriate sign language is displayed from the dataset in the form of images or animations. Machine translation systems are language translation tools that also are capable to be used as learning tools. The process starts with analyzing the text and checking the text-sign dataset and hence generating the output. We use SiGML for converting preprocessed text to sign language. SiGML stands for Signing Gesture Markup Language. It was developed at the University of East Angelia, UK. It describes the HamNoSys symbols in $\mathrm{XML}$ tags forms. It is clear by $3 \mathrm{D}$ rendering software and could be a lot of tractable type of HamNoSys that's accustomed to generate sign animation. There is a translator that has been written to convert HamNoSys of the given word to its SiGML form. A dataset is maintained that contains all the HamNoSys symbols. The translator matches the entered HamNoSys symbols to those which are stored in the database. There are separate files stored for different SiGML tags. The file is generated with the dot SiGML extension along with the name provided by the user.

\section{vii. Recognized Sign Symbol}

The recognized sign symbol will be displayed in the form of an avatar. The JA Signing Avatars, developed at the University of East Angelia, UK, consists of various avatars that are used to play signs. The SiGML file is accessed in the Player for making the signs according to the input text provided. JA Signing is a synthetic animation system that is written in Java. It considers the appropriate Gestural SiGML as input and produces output in the form of motion data for the avatar. There are many types of JA Signing characters used for sign animations.

Algorithms: The algorithm used to implement the Polarity Detection is the SVM algorithm. It consists of three steps: Initialize, Train and Classify

\section{Initialize}

1. Begin

2. Read file containing the data instances for training

3. Read file containing the corresponding labels

4. Convert all data instances to lowercase

5. Remove all punctuation marks and other non-alphanumeric characters from the data instance

6. Perform tokenization, stemming and lemmatization to the input text

7. Split each data instance to its constituent words and remove stop words

8. End

Train the algorithm

1. Begin
2. Loop through each data instance and label pair in training data

3. FOREACH word $w$ in the data instance

4. IF $w$ is not in WordList hash table Then

5. Add $w$ to WordList

6. IF the corresponding label is POSITIVE THEN

7. Set a POSITIVE value of $w$ to 1 and a NEGATIVE value to 0 and NEUTRAL value to 0

8. ELSE

9. Set a POSITIVE value of $w$ to 0 and a NEGATIVE value to 1 and NEUTRAL value to 0

10. ELSE

11. Set a POSITIVE value of $w$ to 0 and a NEGATIVE value to 0 and NEUTRAL value to 1

12. END IF

13. ELSE

14. IF the corresponding label is POSITIVE THEN

15. Add 1 to the POSITIVE value of $w$

16. ELSE

17. Add 1 to the NEGATIVE value of $w$

18. ELSE

19. Add 1 to the NEUTRAL value of $w$

20. End If

21. End If

22. End For

23. End Loop

24. End

25.

Classify sign language

1. BEGIN

2. LOOP while user wants to classify data

3. Read data entered by the user

4. Preprocess the data as in the training process

5. Compute the sum of the POSITIVE, NEGATIVE and NEUTRAL values of the words in the data instance

6. Let these sums be sum1 and sum2 respectively

7. IF sUm $1>$ sUm 2 THEN

8. Classify data instance as belonging to POSITIVE by assigning the appropriate label

9. ELSE IF sum $2<$ sum 1 THEN

10. Classify data instance as belonging to NEGATIVE by assigning the appropriate label

11. ELSE

12. Classify data instance as belonging to NEUTRAL by assigning the appropriate label

13. END IF

14. Add information about the newly classified data instance and its label to the hash table

15. Write the newly classified data instance and its corresponding label to disk

16. END LOOP

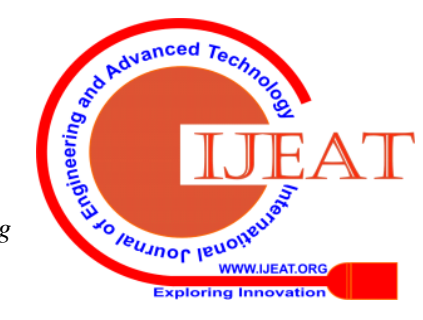




\section{RESULTS AND DISCUSSION}

Initially, the system was checked to predict if the sign languages displayed were accurate according to the input provided. It had shown accurate results for the speech input provided and simultaneously the signs were also displayed by the avatar.

The ISL text is also displayed on the UI, to show the difference between the ISL grammar and the normal English grammar. Since it follows Subject-Object-Verb, the order of the ISL text will differ from the recognized text. Then the polarity detection has been implemented using the SVM machine learning algorithm. There will be three types of outcomes: positive, negative and neutral texts. The next thing to analyze is the speed at which the avatar responds to the pre-processed text. In this way, the avatar would be able to load at a faster rate, and thus increases efficiency. The polarity detection based on the SVM algorithm is used for sentimental analysis.

To train and predict the model, few calculations are involved. The precision can be calculated as shown below:

$$
\text { precision }=\frac{T P}{T P+F P}
$$

Precision can be defined as the measure of correctness that is adapted for accuracy and exactness. It gives refinement in measurement, which can be represented by the number of digits obtained. It is also called as the positive predictive value. Here TP represents true positive and FP represents false positive.

Eq. (1) depicts that for sentences that are correctly classified, TP is used. And for those sentences, which are wrongly classified, FP is used.

$$
\text { recall }=\frac{T P}{T P+F N}
$$

The recall is used to measure the completeness or sensitivity of a classifier. Greater the recall value, lower will be the false negative ad vice-versa. It is measured by the number of true positives divided by some true positives plus the number of false negatives.

In Eq. (2), FN is used for non-classified sentences and TP is for correctly classified sentences.

$$
f \text {-measure }=\frac{\text { precision } X \text { recall } X 2}{\text { precision }+ \text { recall }}
$$

We can obtain the results by measuring in terms of precision, recall, and $f$-measure, as shown in Eq. (3). Using the f-measure value, accuracy can be predicted. The F-score can be defined as the weighted harmonic mean of the test's precision and recall. Table 1 shown below gives an understanding of the actual training and prediction of the model using the specified dataset:

Table 1. Prediction of the model

\begin{tabular}{|c|c|c|c|c|}
\hline Polarity & Precision & Recall & F1-Score & Support \\
\hline Negative & 0.85 & 0.91 & 0.88 & 2327 \\
\hline Neutral & 0.65 & 0.57 & 0.61 & 772 \\
\hline Positive & 0.78 & 0.66 & 0.72 & 561 \\
\hline $\begin{array}{c}\text { Total } \\
\text { Support }\end{array}$ & 3660 & \multicolumn{3}{|l}{} \\
\cline { 1 - 2 } Accuracy & 0.80 & \multicolumn{4}{|l}{} \\
\cline { 1 - 2 } & & &
\end{tabular}

From the table above, the precision, recall and f-measure of the three different types of statements i.e. positive, negative and neutral are mentioned. Thus, we can see that there is an accuracy of $80 \%$ after performing the calculations and also after measuring it with different test cases. The results can further be explained pictorially with the help of the screenshots as shown below:

Fig. 2 demonstrates the UI of the system developed. A web application was created to make it user-friendly.

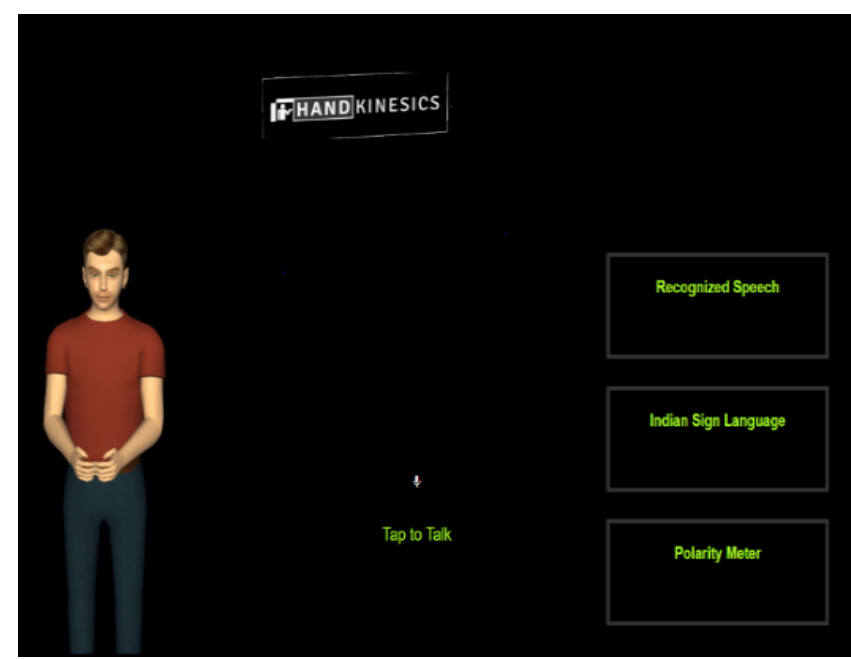

Figure.2 Hand Kinesics in Indian Sign Language Convertor

To check the accuracy, the application was tested with multiple test cases. The corresponding sign languages along with the polarity detection was needed to be checked when the speech input was provided. Also, the text will get converted to the Indian Sign Language text, according to the ISL grammar format.

Testcase 1: To check if the given positive speech input is predicted correctly and if the sign language is depicted accordingly

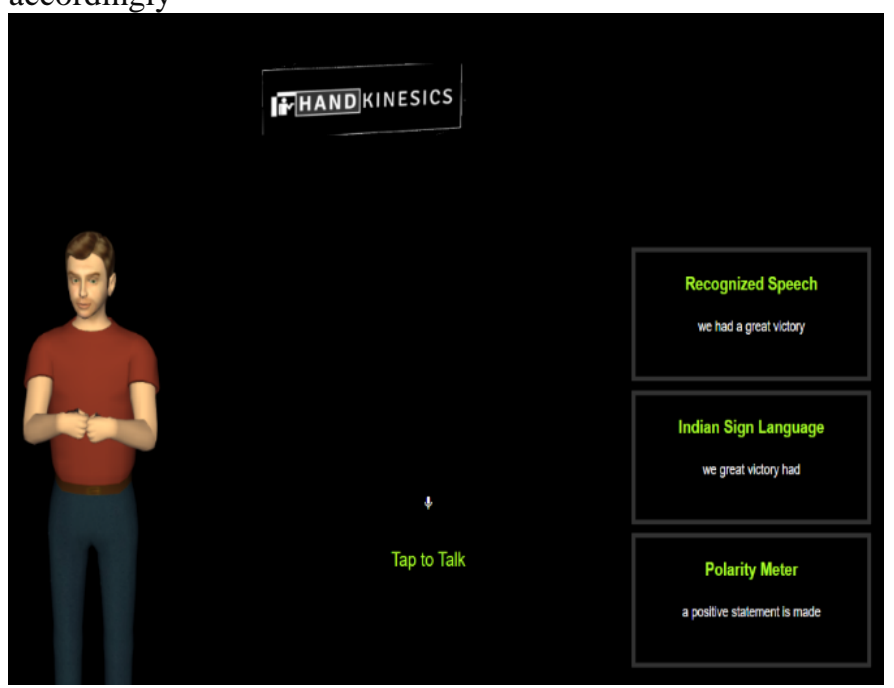

Figure.3 Case 1: Input speech: 'we had a great victory'

In Fig. 3, we can see that for the input text 'we had a great victory', the ISL text was generated according to the ISL grammar rules:

ISL text: 'we great victory had'

Published By:

Blue Eyes Intelligence Engineering

Copyright: All rights reserved.

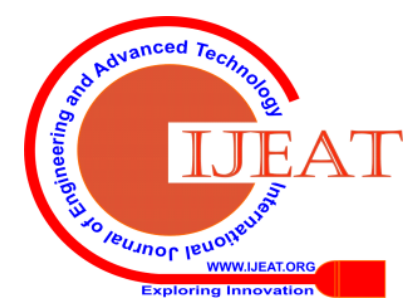




\section{Hand Kinesics in Indian Sign Language using NLP Techniques with SVM Based Polarity}

Also, the polarity meter was depicted as positive for the above statement. The results that are obtained from the application will determine how efficient the application is going to be.

Similarly, we can check the prediction and accuracy with more test cases as shown in Fig. 4, Fig. 5 and Fig. 6.

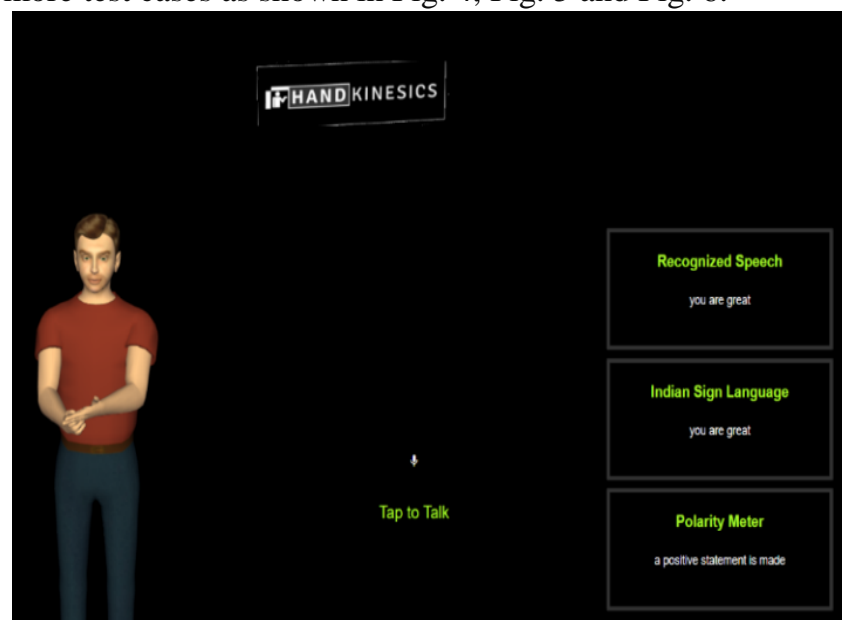

Figure.4 Case 2: Input speech: 'you are great'

Testcase 2: In Fig. 4, the output text is depicted positive and is printed as:

\section{ISL text: 'you are great'}

Testcase 3: Fig. 5 gives the output as a negative statement and displays the ISL text as:

\section{ISL text: 'we match lost'}

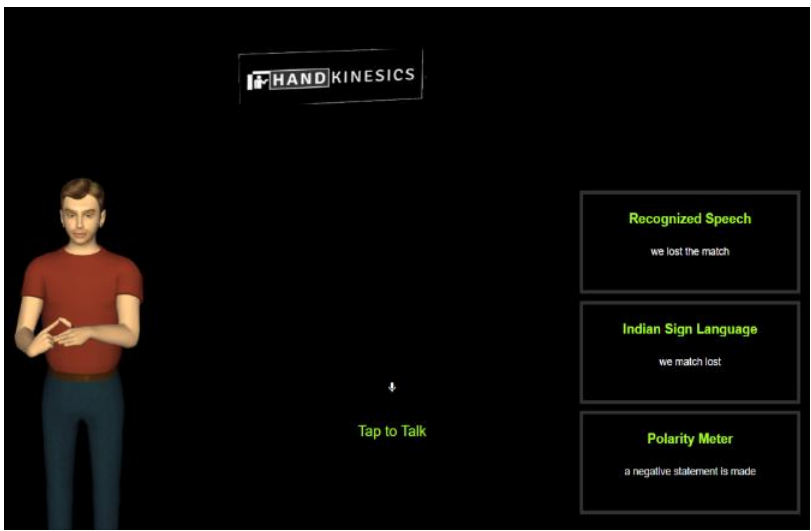

Figure.5 Case 3: Input speech: 'we lost the match'

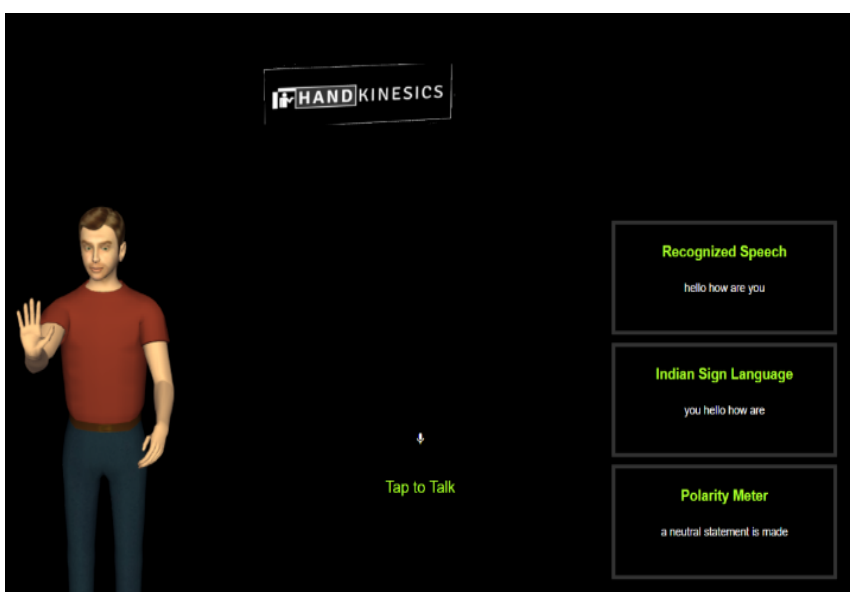

Figure.6 Case 4: Input speech: 'hello how are you'
Testcase 4: In Fig. 6, a neutral statement is made and the corresponding ISL text will be:

\section{ISL text: 'you hello how you'}

Now based on the trained model, we can give it new sentences and the model will be able to classify them based on the sentiment conveyed. It would make more sense to stream sentences regarding casual conversations since our model is trained on such data but trying on random tweets also could help us understand how well our model generalizes in a real-world scenario. It is observed that the SVM algorithm provides the highest accuracy among the other supervised learning algorithms, for small datasets. Thus, this algorithm has proved to be the most efficient one for our problem statement.

The evaluation design was performed where end-users and developers work together to create an evaluation plan. This plan includes the main characteristics of the testing users, the scenarios to simulate and the objective and subjective measurements. The evaluation was carried out. The system was evaluated by a few users who were familiar with the usage of computers. Initially, the system was checked to predict if the sign languages displayed were accurate according to the input provided. The next thing to analyze is the speed at which the avatar responds to the preprocessed text. This is where Machine Learning comes to use. The words spoken by the speaker can be classified and categorized using the SVM algorithm so that the texts are divided into different topics for accuracy and speed of implementation. In this way, the avatar would be able to load at a faster rate, and thus increases efficiency. The figure shown below describes how the SVM algorithm works for positive, negative and neutral statements. In the graph, positive is represented as blue, negative is represented as red and the neutral is defined in the middle i.e. the value of neutral will be positive $=0.5$ and negative $=0.5$. The neutral statements will be displayed in the middle, as shown in the graph.

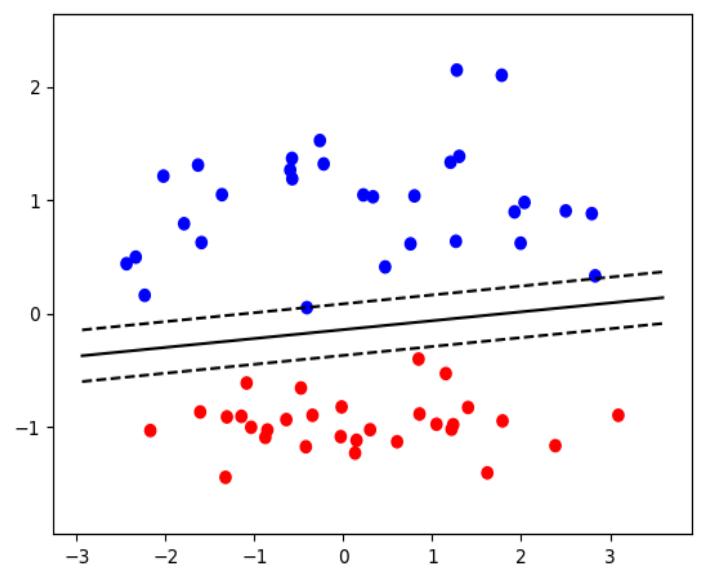

Figure 7. Polarity detection using SVM Algorithm

\section{CONCLUSION}

Published By:

Blue Eyes Intelligence Engineering 
In this project, an efficient speech to Indian Sign Language Translator has been proposed and implemented successfully. This will help mute or deaf people to talk well with normal people and vice-versa.

This research paper creates a system especially for India, to help people easily communicate with mute people using any of the Indian languages as input and provides the appropriate Indian Sign Language (ISL). ISL is a complete natural language and has its grammar and syntax. In this process, it is very important to deal with a good methodology considering the main aspects.

The machine-learning algorithm that has been used here is the Support Vector Machine (SVM) algorithm for polarity detection. It helps to determine whether the input speech provided is a positive, negative or neutral statement. Data sets were used as the input that has been taken from the HamNoSys notation system so as to display the converted audio into the sign language. Also, the polarity detection was performed for sentimental analysis. In this way, we could use the speech data for research purpose; by segregating the speech text in 3 different states: positive, negative and neutral. From the results obtained, we can see that there is a fair accuracy of $80 \%$ (as shown in the table).

During the process of the development of the system, the problem statement was listed out, identified, and addressed systematically. Also, various research and surveys were carried out to study the different methodologies used. Accordingly, the application was designed and developed to meet the goals and requirements. These results determined how efficient the application is going to be. A web application has also been created to make it more user-friendly. Thus, it focuses to make the communication between ordinary people and the hard hearing/mute people easier.

\section{REFERENCES}

1. Jose, M. Jerin, V. Priyadharshini, M. Suresh Anand, and Dr. N. Mohan Kumar" Indian sign language (ISL) translation system for sign language learning." International journal of innovative research and development, vol.2,Issue 5,pp.358-367,2013.

2. López-Ludeña, Verónica, Rubén San Segundo, Carlos González-Morcillo, Juan Carlos López, and E. Ferreiro. "Adapting a speech into sign language translation system to a new domain." In Interspeech, pp. 1164-1168. 2013.

3. Foong, Oi Mean, Tang Jung Low, and Wai Wan La. "V2S: Voice to Sign Language Translation System for Malaysian Deaf People." In International Visual Informatics Conference, Springer, Berlin, Heidelberg, pp. 868-876, 2009.

4. EI-Darymli, Khalid Khalil, Othman O. Khalifa, and Hassan Enemosah. "Speech to Sign Language Interpreter System (SSLIS)" The IEEE International Conference of Computer and Communication Engineering (ICCCE'06), 2006.

5. Szarkowska, Agnieszka. "Text-to-speech audio description: towards wider availability of AD." The Journal of Specialised Translation,

6. MacSweeney, Mairéad, Bencie Woll, Ruth Campbell, Philip K. McGuire, Anthony S. David, Steven CR Williams, John Suckling, Gemma A. Calvert, and Michael J. Brammer. "Neural systems underlying British Sign Language and audio-visual English processing in native users." Brain, vol.125, no. 7, pp.1583-1593, 2002.

7. Sugandhi, Parteek Kumar, Sanmeet Kaur "Online Multilingual Dictionary Using Hamburg Notation for Avatar-Based Indian Sign Language Generation System", International Journal of Cognitive and Language Sciences, vol.12, no.8, 2018

8. Loke, Pranali, Juilee Paranjpe, Sayli Bhabal, and Ketan Kanere. "Indian sign language converter system using an android app." In 2017 International conference of Electronics, Communication and Aerospace Technology (ICECA), vol. 2, pp. 436-439. IEEE, 2017. vol.15, pp.142-162, 2011

9. Arsan, Taner, and Oğuz Ülgen. "Sign language converter." International Journal of Computer Science \& Engineering Survey (IJCSES), vol.6, no. 4, pp.39-51, 2015.

10. AAnand, M. Suresh, A. Kumaresan, and N. Mohan Kumar. "An Integrated Two Way ISL (Indian Sign Language) Translation System--A New Approach." International Journal of Advanced Research in Computer Science, vol.4, no. 1, 2013.

11. DDreuw, Philippe, David Rybach, Thomas Deselaers, Morteza Zahedi, and Hermann Ney. "Speech recognition techniques for a sign language recognition system." In Eighth Annual Conference of the International Speech Communication Association, 2007.

12. GGayathri, A., and A. Sasi Kumar. "Sign Language Recognition for Deaf and Dumb People Using Android Environment." INTERNATIONAL JOURNAL OF CURRENT ENGINEERING AND SCIENTIFIC RESEARCH (IJCESR), vol.4, 2017.

13. PPatil, Gaurangi, Varsha Galande, Vedant Kekan, and Kalpana Dange "Sentiment analysis using support vector machine." International Journal of Innovative Research in Computer and Communication Engineering, vol.2, no. 1, pp. 2607-2612, 2014.

14. ZZainuddin, Nurulhuda, and Ali Selamat. "Sentiment analysis using support vector machine." International Conference on Computer, Communications, and Control Technology (I4CT), pp. 333-337, 2014.

15. SSan-Segundo, Rubén, R. Barra, R. Córdoba, L. F. d'Haro, F. Fernández, Javier Ferreiros, Juan Manuel Lucas, Javier Macías-Guarasa, Juan Manuel Montero, and José Manuel Pardo. "Speech to sign language translation system for Spanish." Speech Communication, vol. 50, no. 11-12, pp.1009-1020, 2008.

16. LLópez-Ludeña, Verónica, Rubén San-Segundo, Carlos González, and Juan Carlos. "Methodology for developing a Speech into Sign Language Translation System in a New Semantic Domain.", 2011.

17. KKaur, Sandeep, and Maninder Singh. "Indian Sign Language animation generation system." 1st International Conference on Next Generation Computing Technologies (NGCT), pp. 909-914. IEEE, 2015.

18. Manoharan, R. Kavitha, A. Pavithra, Meenatchi "Smart Phone-Based Indian Sign Language Maker for Aiding Deaf and Dumb People" International Journal of Research and Scientific Innovation (IJRSI), Vol. 1, Issue 5, 2014

19. PPooja Balu Sonawane, Anita Nikalje "Text to Sign Language Conversion by Using Python and Database of Images and Videos" International Journal of Engineering Research in Electronics and Communication Engineering (IJERECE), Vol.5, Issue 2, 2018

\section{AUTHORS PROFILE}

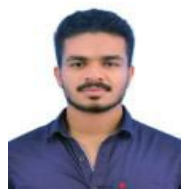

Haren Amal is currently pursuing B.Tech in Computer Science and Engineering at CHRIST (Deemed to be University) India. His research interests are image processing, Machine learning, Speech recognition and Network Security.

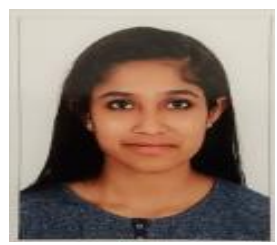

Reena Ann Reny is a B.Tech student in Computer Science and Engineering at CHRIST (Deemed to be University) India. Her research interests image processing, Machine learning, Speech recognition and Network Security.

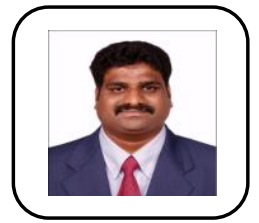

Prathap Rudra Boppuru is an Assistant Professor and Research Scholar in Computer Science and Engineering at CHRIST (Deemed to be University) India. He has completed his B.Tech in Andhra University and M.Tech in JNTUK University. Prathap writes Research articles on crime and analytics, which, considering where you're reading this, makes perfect sense in an Indian context. He has published articles on Crime analysis in Indian context using Social media data.words. 